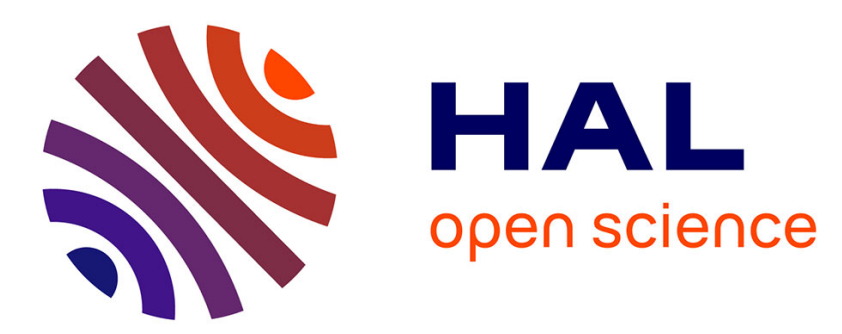

\title{
Mesure de la densité électronique et écarts à l'équilibre de composition dans les arcs de SF6-N2
}

\author{
Manitra Razafinimanana, A. Gleizes, S. Vacquie
}

\section{To cite this version:}

Manitra Razafinimanana, A. Gleizes, S. Vacquie. Mesure de la densité électronique et écarts à l'équilibre de composition dans les arcs de SF6-N2. Revue de Physique Appliquée, 1988, 23 (7), pp.1325-1331. 10.1051/rphysap:019880023070132500 . jpa-00245948

\section{HAL Id: jpa-00245948 https://hal.science/jpa-00245948}

Submitted on 1 Jan 1988

HAL is a multi-disciplinary open access archive for the deposit and dissemination of scientific research documents, whether they are published or not. The documents may come from teaching and research institutions in France or abroad, or from public or private research centers.
L'archive ouverte pluridisciplinaire HAL, est destinée au dépôt et à la diffusion de documents scientifiques de niveau recherche, publiés ou non, émanant des établissements d'enseignement et de recherche français ou étrangers, des laboratoires publics ou privés. 


\title{
Mesure de la densité électronique et écarts à l'équilibre de composition dans les arcs de $\mathrm{SF}_{6}-\mathrm{N}_{2}$
}

\author{
M. Razafinimanana, A. Gleizes et S. Vacquie \\ Laboratoire des décharges dans les gaz, UA n ${ }^{\circ}$ 277, CPAT, Université Paul Sabatier, 118 route de Narbonne, \\ 31062 Toulouse Cedex, France
}

(Reçu le 3 février 1988, révisé le 14 mars 1988, accepté le 24 mars 1988)

\begin{abstract}
Résumé. - La densité électronique a été mesurée dans des arcs stabilisés brûlant dans des mélanges $\mathrm{SF}_{6}-\mathrm{N}_{2}$, par des techniques de spectroscopie d'émission et d'interférométrie laser à deux longueurs d'onde. Les variations relatives de la densité électronique en fonction du pourcentage de $\mathrm{SF}_{6}$ dans le mélange et en fonction de l'intensité du courant sont en bon accord avec les variations calculées par un modèle supposant l'équilibre thermique. Cependant l'étude des valeurs réelles de la densité électronique en fonction des valeurs mesurées de la température a permis de mettre en évidence des écarts à la composition d'équilibre du plasma. On montre que lorsque la proportion de $\mathrm{SF}_{6}$ est supérieure à $10 \%$, ces écarts sont essentiellement dus au phénomène de démixion entre le fluor et le soufre.
\end{abstract}

\begin{abstract}
The electron number density $n_{\mathrm{e}}$ has been measured in wall-stabilized arcs burning in $\mathbf{S F}_{6}-\mathrm{N}_{2}$ mixtures, by spectroscopy and by two-wavelength laser interferometry. The relative variations of the electron density versus $\mathrm{SF}_{6}$ proportion and versus current intensity are in good agreement with the variations calculated by a modeling assuming thermal equilibrium. Nevertheless the real variations of $n_{\mathrm{e}}$ in function of the measured values of the temperature show departures from the plasma composition. When the proportion of $\mathrm{SF}_{6}$ is greater than $10 \%$ these departures are mainly due to demixing effect between fluorine and sulfur.
\end{abstract}

\section{Introduction.}

Dans un article précédent [1], nous avons rapporté les valeurs mesurées et calculées de la température dans un plasma d'arc stabilisé par paroi brûlant dans des mélanges $\mathrm{SF}_{6}-\mathrm{N}_{2}$ à la pression atmosphérique. Nous avons montré que dans ce type de plasma les variations mesurées et calculées de la température en fonction de l'intensité du courant et de la proportion de chaque constituant dans le mélange, sont analogues. Ceci nous avait permis de dégager le comportement général d'arcs brûlant dans des mélanges en fonction des propriétés de transport (conductivités électrique et thermique, rayonnement) des gaz formant ce mélange. Toutefois, à la fin de cet article, nous avons indiqué que certaines différences entre expérience et théorie pouvaient être dues à l'existence d'écarts à l'équilibre thermodynamique local (ETL). C'est ce point particulier que nous aborderons ici.

Dans un plasma thermique, les écarts à l'équilibre thermodynamique local peuvent avoir plusieurs origines :
- La fonction de distribution des vitesses des particules n'est pas maxwellienne. La notion de température perd alors son sens habituel et les formules caractéristiques de l'ETL ne sont pas applicables.

- La diffusion ambipolaire conduit à un souspeuplement en électrons de la région axiale par rapport à la densité électronique d'équilibre calculée au moyen de la température du plasma. On a alors $n_{\mathrm{e}_{\text {calcule }}} \neq n_{\mathrm{e}_{\text {exp }}}$.

- Le transfert d'énergie des électrons vers les particules lourdes n'est pas suffisant pour amener la température des ions et des atomes $T_{\mathrm{g}}$ à une valeur voisine de celle des électrons $T_{\mathrm{e}}$. On a alors $T_{\mathrm{e}} \neq T_{\mathrm{g}}$.

- Les désexcitations radiatives des atomes ne sont pas négligeables devant les transferts d'énergie collisionnels. Les formules de Boltzmann et de Saha ne sont plus applicables. (Elles peuvent l'être partiellement par rapport à un niveau excité.)

- Dans le cas d'un mélange de gaz ou d'un gaz 
moléculaire composé d'atomes différents, les phénomènes de diffusion peuvent modifier le rapport stœchiométrique des concentrations théoriques de chaque constituant (démixion). La loi de GuldbergWaage est alors mise en défaut et la composition du plasma ne peut être calculée.

C'est essentiellement sur ce dernier type d'écart que portera ici notre étude. Nous avons mesuré la densité électronique et étudié les déviations à l'équilibre de composition en comparant les valeurs mesurées de différentes grandeurs. Les valeurs expérimentales sont comparées aux valeurs d'équilibre et à celles obtenues à partir d'une modélisation de la décharge.

Pour mettre en évidence des écarts à l'équilibre, on utilise généralement deux méthodes. La première consiste à mesurer des grandeurs en dehors de toute hypothèse d'équilibre (en général des densités de particules) et à comparer les valeurs des paramètres déduits de ces grandeurs aux valeurs des paramètres à l'équilibre. La deuxième consiste à déterminer des valeurs de températures correspondant à différentes lois de l'équilibre et à comparer leurs valeurs : température d'excitation par un diagramme de Boltzmann, température d'ionisation par le rapport des intensités des raies de deux ions différents, températures de rotation et de vibration des molécules, etc. Les différences sensibles entre elles indiquent des écarts à l'équilibre. Dans notre cas nous allons tout d'abord comparer des variations de densités de particules puis nous étudierons les corrélations entre la densité électronique et la "température " définie à partir de la mesure de l'intensité de deux raies (de $\mathrm{S}$ et de $\mathrm{S}^{+}$). Les mesures sont relatives à la zone axiale de la décharge qui est la région la plus chaude. Les molécules non dissociées y sont en densité négligeable.

\section{Mesure de la densité électronique.}

Un des arcs stabilisés étudiés a été décrit en détail dans la référence [1]. Il correspond à un diamètre d'arc de $4 \mathrm{~mm}$. Pour d'autres expériences, nous avons utilisé un arc de diamètre $5 \mathrm{~mm}$ dont la conception légèrement différente a été décrite dans la référence [2]. Dans les deux cas, les électrodes de tungstène sont protégées de l'érosion chimique du $\mathrm{SF}_{6}$ par un jet d'argon.

Deux techniques de diagnostic ont été développées : la spectroscopie d'émission et l'interférométrie laser à deux longueurs d'onde. Les dispositifs correspondants sont respectivement détaillés dans les références [1] et [2].

Pour obtenir les valeurs de la densité électronique, deux approches ont été réalisées. $\mathrm{La}$ première consiste à utiliser des méthodes ne nécessitant pas d'hypothèses sur l'état d'équilibre du plasma. La deuxième consiste à déduire la densité électronique de la valeur mesurée de la température.

\subsection{MESURES SANS HYPOTHÈSE D'ÉQUILIBRE SUR LE PLASMA.}

Méthodes. - Trois méthodes ont ainsi été développées pour mesurer la densité électronique $n_{\mathrm{e}}$. La première est l'interférométrie laser à deux longueurs d'onde : le principe de la méthode, le montage et certains résultats ont été donnés précédemment [2]. Rappelons brièvement que cette méthode consiste à mesurer les variations de la réfractivité du plasma durant son extinction, pour deux valeurs de la longueur d'onde. Compte tenu de la dépendance de la réfractivité envers les densités de particules et envers la longueur d'onde [2,3], on peut déduire de cette mesure les variations de la densité électronique durant l'extinction. Les résultats ainsi obtenus sont indépendants de toute hypothèse concernant l'état d'équilibre du plasma. Dans cette expérimentation le plasma d'argon situé au voisinage des électrodes perturbe la mesure. Il faut l'éliminer. Pour cela, lorsque l'arc est stabilisé on supprime l'argon, puis on attend quelques secondes ou quelques dizaines de secondes pour que le $\mathrm{SF}_{6}$ occupe tout le volume de la chambre (vérification avec la tension d'arc). Alors on déclenche l'extinction de l'arc. On s'assure ainsi d'une coupure dans le $\mathrm{SF}_{6}$ pur en limitant l'usure des électrodes et le dégagement de "fumées " qui en découle.

La deuxième méthode est fondée sur la mesure de l'élargissement Stark de la raie 493,5 nm de NI. On utilise les résultats de Helbig et al. [4], Baronnet et al. [5] et Bacri et Lagreca [6] reliant les valeurs de cet élargissement à celles de la densité électronique. La procédure expérimentale consiste à enregistrer le profil de la raie par visée longitudinale et à déconvoluer ce profil par la fonction d'appareil. En pratique on utilise la relation donnée par Van de Hulst et Reesink [7] pour éliminer du profil de Voigt mesuré la contribution due à la fonction d'appareil. Cette fonction est assimilée à une gaussienne dont la largeur à mi-hauteur est $0,026 \mathrm{~nm}$ dans nos conditions expérimentales.

La troisième méthode est caractéristique d'un plasma contenant du soufre : nous avons montré [8] que la densité électronique peut être déduite de la forme du triplet $469,5 \mathrm{~nm}$ de SI. Le rapport entre la valeur du maximum de la raie la plus intense et la valeur du minimum relatif entre deux raies de ce triplet est une fonction monotone et sensible de la densité électronique dans la gamme $2 \times 10^{16-7} \times$ $10^{16} \mathrm{~cm}^{-3}$.

Remarquons que contrairement au cas de la mesure par interférométrie laser, les résultats des deux dernières techniques ne sont pas perturbés par la présence d'argon au niveau des électrodes puisqu'on mesure des grandeurs caractéristiques du mélange $\mathrm{SF}_{6}-\mathrm{N}_{2}$ (la longueur de la colonne de plasma de $\mathrm{SF}_{6}-\mathrm{N}_{2}$ étant déterminée avec précision, cf. [1]) 


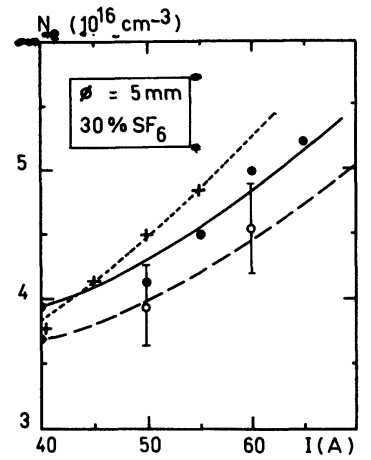

Fig. 1. - Variations mesurées de la densité électronique sur l'axe en fonction de l'intensité du courant : + méthode du triplet ; • élargissement Stark ; O interférométrie laser.

[Electron density versus current intensity, along the axis : + triplet method; - Stark broadening; O laser interferometry.]

Résultats. - Des résultats typiques sont représentés sur la figure 1 ; il s'agit des variations de la densité électronique $n_{\mathrm{e}}$ mesurées sur l'axe de la décharge, en fonction de l'intensité du courant pour un diamètre d'arc de $5 \mathrm{~mm}$ et un mélange contenant $30 \%$ de $\mathrm{SF}_{6}$ et $70 \%$ d'azote, en volume. Les valeurs les plus précises sont celles obtenues par interférométrie laser avec une incertitude de $10 \%$. Les valeurs déduites de l'élargissement de la raie $493,5 \mathrm{~nm}$ de NI sont légèrement supérieures mais en bon accord. Les valeurs de $n_{\mathrm{e}}$ déduites de la méthode du triplet de SI à $\lambda=469,5 \mathrm{~nm}$ sont également en accord lorsque l'intensité du courant est inférieure à $60 \mathrm{~A}$. Au-delà, cette méthode donne des résultats divergents par rapport aux autres méthodes. Cela correspond assez bien aux limitations de la méthode [8] qui n'est plus applicable lorsque $n_{\mathrm{e}} \geqq 6 \times$ $10^{16} \mathrm{~cm}^{-3}$, l'écart entre le maximum et le minimum relatif du profil d'intensité devenant trop faible. Dans la discussion sur les écarts à l'équilibre nous utiliserons comme valeurs de la densité électronique, celles déduites de l'interférométrie ou de l'élargissement Stark de la raie d'azote. Sur la figure 2 sont indiquées les variations de la densité électronique mesurée par interférométrie laser en fonction de l'intensité du courant et pour différentes proportions du mélange $\mathrm{SF}_{6}-\mathrm{N}_{2}$. Nous reviendrons sur ces résultats dans la partie 2.2.

\subsection{Mesure Couplée AVEC CELle DE LA TEMPÉ-} RATURE. - Dans le cas des plasmas d'arc stationnaires dans le $\mathrm{SF}_{6}$ pur, une des principales causes d'écart à l'équilibre est la démixion $[8,9]$. La démixion est une combinaison de la diffusion et des mécanismes d'ionisation dans des mélanges qui induisent un déséquilibre chimique dans le plasma. Dans le cas du $\mathrm{SF}_{6}$, la démixion se traduit sur l'axe d'un arc par un appauvrissement relatif en soufre : le

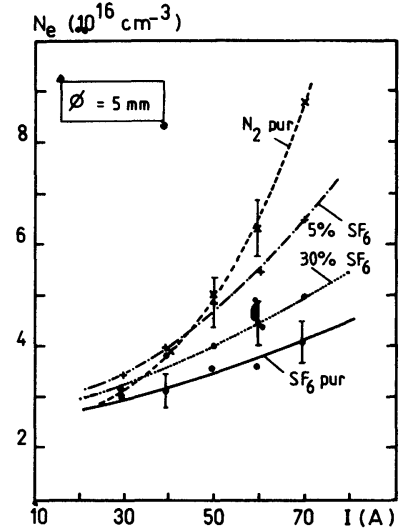

Fig. 2. - Variations de la densité électronique mesurée par interférométrie laser pour différentes proportions du mélange $\mathrm{SF}_{6}-\mathrm{N}_{2}$.

[Electron density measured by laser interferometry for several $\mathrm{SF}_{6}-\mathrm{N}_{2}$ mixtures.]

rapport des concentrations de fluor et de soufre $M=|\mathrm{F}| /|\mathrm{S}|$ est supérieur à 6 . A partir de cette constatation, et en suivant l'idée de Schulz-Gulde [9] pour le $\mathrm{SF}_{6}$ pur, nous avons mis au point une mesure de température dans le plasma d'arc de $\mathrm{SF}_{6}-\mathrm{N}_{2}$. Cette méthode a été décrite en détail dans la référence [1]. Rappelons-en brièvement le principe :

- dans un plasma en équilibre, à une pression donnée, la connaissance d'une seule grandeur locale (température ou densité) permet de déterminer toutes les autres;

- dans notre cas, nous supposons que toutes les lois de l'équilibre thermodynamique local sont réalisées sauf celle de la dissociation du $\mathrm{SF}_{6}$ (le rapport $M$ précédemment défini n'est pas connu $a$ priori). Dans ces conditions il faut mesurer deux paramètres pour obtenir tous les autres.

Nous avons choisi de mesurer l'intensité absolue de deux raies, l'une de SI, à 469,5 nm et l'autre de SII à $545,4 \mathrm{~nm}$. Ce choix permet d'avoir une assez bonne précision sur $T$ car les lois de Saha et de Boltzmann s'appliquent mieux au soufre qu'au fluor compte tenu des structures atomiques de ces deux espèces. Dans la référence [1] nous avons donné des valeurs de température déduites de cette mesure. On a vu que l'application des lois de l'équilibre permet d'obtenir les valeurs des autres paramètres tels que le rapport $M$ ou la densité electronique. Nous présentons sur la figure 3 les variations de la densité électronique sur l'axe de la décharge, obtenues par cette méthode, pour un arc de $4 \mathrm{~mm}$ de diamètre, en fonction de l'intensité du courant. Ces résultats sont tout à fait cohérents avec ceux de la figure 2 : variations analogues suivant la proportion de $\mathrm{SF}_{6}$ (la méthode indirecte ne permet pas d'obtenir les valeurs de $n_{\mathrm{e}}$ dans un plasma d'azote pur); valeurs supérieures pour un arc de $4 \mathrm{~mm}$ de diamètre 


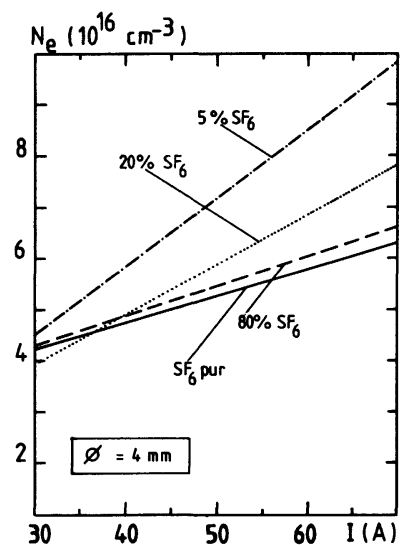

Fig. 3. - Variations de la densité électronique déduites de la mesure des intensités absolues des raies de $\mathrm{S}$ et $\mathrm{S}^{+}$

[Electron density deduced from the measurements of absolute intensities of $\mathrm{S}$ and $\mathrm{S}^{+}$lines.]

par rapport à celles d'un arc de $5 \mathrm{~mm}$ de diamètre (la diminution du diamètre de stabilisation entraîne de plus forts gradients et des valeurs axiales plus élevées).

Remarque. - Lorsque la proportion d'azote dans le mélange est supérieure à $20 \%$ nous avons aussi couplé la mesure de l'intensité absolue de la raie $493,5 \mathrm{~nm}$ de NI à celle des raies de SI et SII pour obtenir une valeur de $T$ indépendante du rapport effectif d'azote sur $\mathrm{SF}_{6}$ [1]. Cette méthode analogue dans son principe à celle indiquée ici conduit à des résultats très peu différents (moins de $100 \mathrm{~K}$ d'écart sur la température).

\section{Calcul de la densité électronique.}

Le calcul est basé sur l'équation du bilan d'énergie d'un arc stationnaire en équilibre thermique, qui s'écrit en coordonnées cylindriques [1] :

$$
\sigma E^{2}+\frac{1}{r} \frac{\partial}{\partial r}\left(r \kappa \frac{\mathrm{d} T}{\mathrm{~d} r}\right)-u=0 .
$$

Les fonctions matérielles, $\sigma$ (conductivité électrique), $\kappa$ (conductivité thermique) et $u$ (rayonnement), ont été précédemment calculées pour un plasma en équilibre. La résolution de l'équation (1) permet d'obtenir les variations de température. En supposant que le plasma est en équilibre, les valeurs de la densité électronique se déduisent des valeurs de la température.

Sur la figure 4 sont représentées les variations ainsi calculées de la densité électronique sur l'axe de la décharge, pour un arc de $4 \mathrm{~mm}$ de diamètre, en fonction de l'intensité du courant. Ces mêmes variations ont été calculées pour un arc de $5 \mathrm{~mm}$ de diamètre ; elles correspondent à des courbes tout à fait analogues à celles de la figure 4 (en particulier croisement des courbes pour une intensité de courant

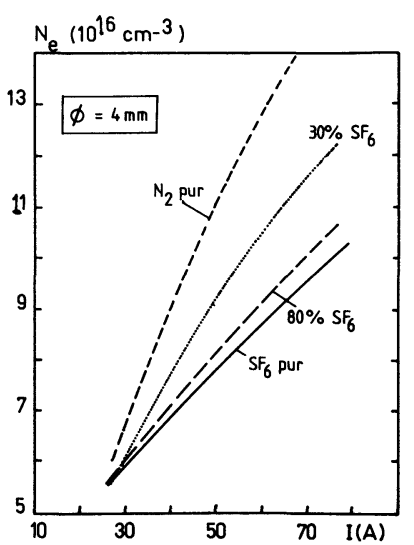

Fig. 4. - Variations calculées de la densité électronique sur l'axe $(\phi=4 \mathrm{~mm})$.

[Calculated variations of the electron density on the discharge axis $(\phi=4 \mathrm{~mm})$.]

comprise entre 25 et $30 \mathrm{~A}$ ), les valeurs de $n_{\mathrm{e}}$ étant plus faibles d'environ $2 \times 10^{16} \mathrm{~cm}^{-3}$ pour une valeur donnée du courant. Si l'on compare cette figure aux variations mesurées représentées sur la figure 3 , on observe un bon accord entre expérience et théorie dans les variations relatives de la densité électronique. Les différences dans les courbures pouvant provenir de l'erreur sur les valeurs expérimentales ou sur l'imprécision des fonctions matérielles (en particulier rayonnement dû à l'azote). Par contre, on observe un désaccord dans les valeurs absolues : les valeurs calculées sont systématiquement supérieures aux valeurs mesurées.

Puisque les valeurs calculées sont fondées sur l'hypothèse de l'équilibre thermique on peut supposer que cette différence expérience-théorie est due à l'existence d'écarts à l'équilibre. Ceci n'est pas certain. En effet, bien que calculées en utilisant une composition d'équilibre du $\mathrm{SF}_{6}$, les fonctions matérielles $\sigma$ et $\kappa$ dépendent de sections efficaces qui ne sont pas connues avec une bonne précision; par ailleurs, le coefficient d'émission net dans les mélanges $\mathrm{SF}_{6}-\mathrm{N}_{2}$ est mal connu. Cette imprécision sur les coefficients de l'équation (1) peut engendrer une erreur systématique sur la température et donc sur la densité électronique qui en est déduite. Pour pouvoir étudier les écarts à l'équilibre, il faut établir des corrélations entre des grandeurs mesurées indépendamment et les comparer aux corrélations à l'équilibre ; c'est ce que nous allons présenter maintenant.

\section{Ecarts à l'équilibre.}

4.1 VARIATIONS DES DENSITÉS EN FONCTION DE LA TEMPÉRATURE. - Nous présentons sur la figure 5 les variations de la densité électronique et de la densité d'ions $\mathrm{S}^{+}$en fonction de la température pour un mélange $80 \% \mathrm{SF}_{6}+20 \% \mathrm{~N}_{2}$. Ces valeurs sont 


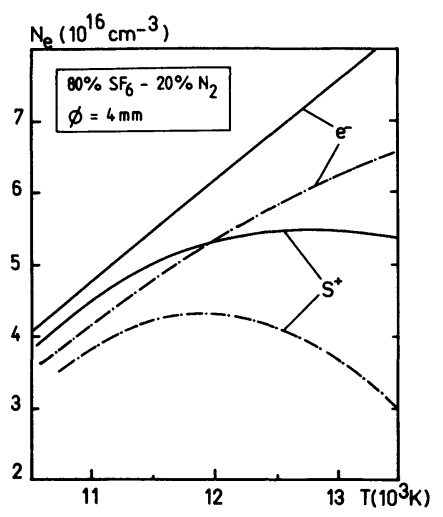

Fig. 5. - Evolutions des densités d'électrons et d'ions $\mathrm{S}^{+}$ avec la température : - valeurs d'équilibre ; - - - valeurs déduites de la mesure des intensités absolues des raies de $\mathrm{S}$ et $\mathrm{S}^{+}$.

[Evolution of the number densities of $\mathrm{S}^{+}$ions and electrons in function of the temperature :- equilibrium values; - - - values deduced from measurements of absolute intensities of $S$ and $\mathrm{S}^{+}$lines.]

toutes déduites de la mesure de l'intensité absolue des deux raies de SI et SII en suivant la procédure décrite au paragraphe 2.2. Deux séries de valeurs sont représentées sur cette figure : celles déduites de l'expérience et celles correspondant à la composition d'équilibre du mélange considéré. On observe un comportement analogue entre ces deux séries. Pour les valeurs les plus faibles de la température, l'ionisation du plasma est due essentiellement au soufre. La densité $n_{\mathrm{S}^{+}}$est donc proche de $n_{\mathrm{e}}$; au fur et à mesure que la température augmente $\left(n_{\mathrm{e}}-n_{\mathrm{S}^{+}}\right)$augmente à cause de l'ionisation de $\mathrm{N}$ puis de $\mathrm{F}$. Par contre, on observe une différence systématique entre les valeurs d'équilibre et les valeurs déduites des mesures. Compte tenu de la méthode utilisée, cette différence ne peut provenir que du déséquilibre de dissociation induit par la démixion.

Pour étudier avec plus de précision ce mécanisme, nous avons porté sur les figures 6 et 7 les variations de la densité électronique en fonction de la température, sur l'axe d'un arc de $4 \mathrm{~mm}$ de diamètre. Sur chaque figure trois courbes sont tracées : la première correspond aux variations de $n_{\mathrm{e}}$ dans un plasma en équilibre ; la deuxième correspond aux variations vraies de la densité électronique (mesurée par élargissement Stark) en fonction de la température mesurée par la méthode du paragraphe 2.2. Enfin la troisième correspond aux variations de $n_{\mathrm{e}}$ déduite de la méthode du paragraphe 2.2 : elles correspondent aux valeurs d'équilibre du plasma $\left(\mathrm{N}_{2}+\mathrm{S}+\mathrm{F}\right)$ dans lequel le rapport $\mathrm{F} / \mathrm{S}$ n'est pas fixé à 6 .

De ces figures 6 et 7 , on peut tirer plusieurs enseignements :

i) Compte tenu des marges d'erreur, on met en évidence des écarts à l'équilibre dans les deux

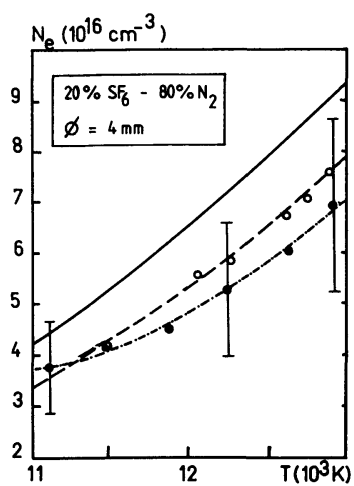

Fig. 6. - Variations de la densité électronique avec la température : — composition à l'équilibre ; • valeurs mesurées par élargissement Stark; O valeurs déduites de la mesure des intensités des raies de $\mathrm{S}$ et $\mathrm{S}^{+}$.

[Variations of electron density with temperature. equilibrium values; • values measured by Stark broadening; $O$ values deduced from the measurements of absolute intensities of $\mathrm{S}$ and $\mathrm{S}^{+}$lines $\left(20 \% \mathrm{SF}_{6}-80 \%\right.$ $\mathrm{N}_{2}$ ).]

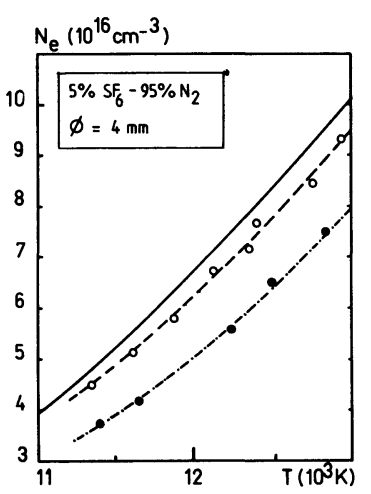

Fig. 7. - Idem que figure 6 pour un mélange à $5 \% \mathrm{SF}_{6^{-}}$ $95 \% \mathrm{~N}_{2}$.

[The same as figure 6 with a mixture containing $5 \% \mathrm{SF}_{6}$ $95 \% \mathrm{~N}_{2}$.]

mélanges étudiés (comparaison de la courbe ETL et des valeurs vraies).

ii) Ces écarts ne diminuent pas quand la température augmente. Ce comportement est différent de celui observé généralement sur l'axe des plasmas d'arc formés par une seule espèce.

iii) La prise en compte de la démixion comme seul phénomène d'écart à l'équilibre permet d'expliquer les résultats pour le mélange à $80 \% \mathrm{~N}_{2}$ et $20 \%$ $\mathrm{SF}_{6}$ mais ne permet pas d'expliquer les écarts observés dans le mélange à $95 \% \mathrm{~N}_{2}+5 \% \mathrm{SF}_{6}$.

4.2 INFLUENCE DE LA PROPORTION DE $\mathrm{SF}_{6}$ SUR LA DÉMIXION. - Pour affiner notre analyse, examinons les valeurs du rapport $M$ entre la concentration totale de fluor (ions + atomes) et la concentration 
Tableau I. - Valeurs du rapport de concentration $M=|\mathrm{F}| /|\mathrm{S}|$ pour différentes proportions $d u$ mélange $\mathrm{SF}_{6}-\mathrm{N}_{2}$.

[Values of the concentration ratio $M=|\mathrm{F}| /|\mathrm{S}|$ for various mixtures $\mathrm{SF}_{6}-\mathrm{N}_{2}$.]

\begin{tabular}{|c|c|c|c|}
\hline$T(\mathrm{~K})$ & $5 \% \mathrm{SF}_{6}$ & $20 \% \mathrm{SF}_{6}$ & $\mathrm{SF}_{6} \mathrm{pur}$ \\
\hline 11250 & 16,4 & 13,8 & 7,7 \\
\hline 11500 & 17 & 14,3 & 8 \\
\hline 12000 & 18,7 & 16,3 & 8,5 \\
\hline 12500 & 20,5 & 19 & 9 \\
\hline
\end{tabular}

totale de soufre (ions + atomes). Ces valeurs, données par la méthode de détermination de la température $T$, sont portées sur le tableau I pour trois types de mélange, en fonction de $T$. Dans la gamme de température étudiée, on observe que $M$ augmente avec la température, et que $M$ augmente également quand la proportion de $\mathrm{SF}_{6}$ diminue. La première tendance est en accord avec le phénomène déjà observé pour le $\mathrm{SF}_{6}$ pur [8,9]. La deuxième est a priori plus étonnante. Elle s'explique par la forme des profils radiaux de température. Dans l'azote, en effet, pour une intensité de courant voisine de $40 \mathrm{~A}$ $(\phi=4 \mathrm{~mm})$ le profil de température est assez abrupt dans la partie centrale puis présente un plateau vers $7500 \mathrm{~K}$. Dans le $\mathrm{SF}_{6}$ la décroissance $T(r)$ est plus douce (cf. Fig. 5 de la référence [1]). Ces caractéristiques sont dues aux variations de la conductivité thermique avec la température. Dans les mélanges $\mathrm{SF}_{6}-\mathrm{N}_{2}$ nous avons montré [1] que plus la proportion de $\mathrm{N}_{2}$ est forte plus le profil est abrupt, ce qui renforce les gradients de densité. Le flux de diffusion ambipolaire, près de l'axe, est alors plus important ce qui accentue les effets de démixion. Le facteur $M$ (déduit des mesures sur l'axe) est donc d'autant plus important que la proportion de $\mathrm{SF}_{6}$ est faible dans le mélange.

Cette propriété semble en contradiction avec les commentaires des figures 6 et 7 dans lesquels nous avons dit que la démixion entre le fluor et le soufre expliquait les écarts à l'équilibre dans un mélange à $20 \% \mathrm{SF}_{6}$ mais ne pouvait rendre compte des écarts dans un mélange à $5 \% \mathrm{SF}_{6}$.

En fait la contradiction n'est qu'apparente : si la démixion est la principale source d'écart à l'équilibre observé sur la densité électronique pour les plasmas de $\mathrm{SF}_{6}$ pur et des mélanges $\mathrm{SF}_{6}-\mathrm{N}_{2}$ contenant $20 \%$ et plus de $\mathrm{SF}_{6}$, ceci n'est plus le cas lorsque la concentration relative de $\mathrm{SF}_{6}$ devient inférieure à $10 \%$. En effet, dans ce cas les électrons du plasma proviennent davantage de l'ionisation de l'azote que de celle du soufre (compte tenu de la faible concentration totale de soufre). Dans ces conditions de faible proportion de $\mathrm{SF}_{6}$, même si la concentration de soufre est fortement perturbée par le mécanisme de démixion (ce qu'indiquent les valeurs élevées de $M$ dans ce cas) ceci n'a qu'une faible influence sur la concentration d'électrons.

4.3 Autres Causes d'ÉCARTS À L'ÉQuilibre. Rappelons tout d'abord que les valeurs de la température déduites des intensités des raies de $\mathrm{S}$ et $\mathrm{S}^{+}$ correspondent à l'hypothèse d'une composition d'équilibre d'un plasma de mélange $\mathrm{S}+\mathrm{F}+\mathrm{N}$, avec un rapport stœchiométrique entre $\mathrm{F}$ et $\mathrm{S}$ différent de 6. Cela veut dire que lorsque la courbe $n_{\mathrm{e}}(T)$ déduite de cette procédure est proche des valeurs expérimentales (et différente de la courbe d'équilibre d'un plasma de $\mathrm{SF}_{6}$ ) la démixion est la cause principale des écarts à l'équilibre de composition (Fig. 6). A contrario, quand cette courbe est proche de la courbe ETL du $\mathrm{SF}_{6}$ et différente de la courbe expérimentale, les écarts à l'équilibre ne sont pas (essentiellement) dus à la démixion (Fig. 7).

Il reste à analyser l'origine de ces écarts à l'équilibre dans ce dernier cas (très faibles proportions de $\mathrm{SF}_{6}$ ). Deux causes peuvent être invoquées :

- phénomènes non réversibles que l'on rencontre dans les plasmas d'azote pur,

- propriétés du mélange et/ou limites de la méthode d'investigation.

Dans la première cause on retrouve l'influence directe de la diffusion ambipolaire qui dépeuple l'axe de la décharge en électrons et dépend directement des profils radiaux de température [10]. Le déséquilibre statistique (écarts aux lois de Saha et Boltzmann pour tous les niveaux des différentes espèces) dû à l'importance des phénomènes radiatifs par rapport aux collisions inélastiques [11, 12], est quantitativement difficile à étudier dans notre cas. En particulier on ne peut définir le point Saha correspondant à l'extrapolation du diagramme de Boltzmann pour une espèce neutre jusqu'à la limite d'ionisation car on n'a pas l'égalité entre la densité électronique et la densité d'une espèce d'ions positifs [13]. D'après le modèle collisionnel radiatif de Taylor et Ali [14] le plasma d'azote pur n'est en ETL complet que pour $n_{\mathrm{e}} \geqq 5 \times 10^{17} \mathrm{~cm}^{-3}$, ce qui n'est pas vérifié dans nos conditions.

Dans la deuxième cause d'écart intervient tout d'abord le mécanisme de démixion de l'azote par rapport au fluor. Mais, compte tenu de la forte concentration d'azote par rapport au fluor (dans un mélange à $95 \% \mathrm{~N}_{2}+5 \% \mathrm{SF}_{6}$ ) et de la différence relativement faible entre les potentiels d'ionisation du fluor et de l'azote, nos résultats montrent que cette démixion a une influence négligeable sur la densité électronique. Pour expliquer les écarts obser- 
vés sur la figure 7 il faut aussi remarquer que le principe de la méthode de détermination des paramètres fondée sur la mesure des intensités de deux raies de SI et de SII, conduit à une bonne précision sur $T$ et sur la densité d'ions $\mathrm{S}^{+}$. Cette précision est liée à la validité de l'équilibre de Boltzmann et à la précision du coefficient d'émission spontanée de la raie de SII. Par contre, cette méthode conduit à une précision médiocre sur $n_{\mathrm{e}}$ dans la mesure où $n_{\mathrm{e}}$ est différent de $n_{\mathrm{S}^{+}}$, ce qui est le cas pour un mélange à $5 \%$ de $\mathrm{SF}_{6}$.

\section{Conclusion.}

Les variations mesurées de la densité électronique et de la température sur l'axe d'un arc brûlant dans les mélanges $\mathrm{SF}_{6}-\mathrm{N}_{2}$ nous ont permis de mettre en évidence des écarts à l'équilibre, bien que le comportement général de ces arcs respectent qualitativement les évolutions prévues par une modélisation fondée sur l'hypothèse d'équilibre thermique. Les écarts à l'équilibre dans ces mélanges, comme dans le $\mathrm{SF}_{6}$ pur, sont dus à la démixion qui, en appauvrissant l'axe de la décharge en soufre, conduit à une sous-population électronique par rapport à la composition d'équilibre.

La démixion entre le soufre et le fluor augmente quand la concentration de $\mathrm{SF}_{6}$ diminue. Toutefois lorsque la concentration de $\mathrm{SF}_{6}$ devient inférieure à $10 \%$ la densité électronique est peu affectée par ce mécanisme de démixion et les principaux écarts à l'équilibre sont alors ceux spécifiques des plasmas d'arc dans l'azote pur.

\section{Bibliographie}

[1] Gleizes, A., Razafinimanana, M., Vacouie, S., Revue Phys. Appl. 22 (1987) 1411.

[2] Gleizes, A., Razafinimanana, M., Vacouie, S., J. Appl. Phys. 54 (1983) 3377.

[3] Ascoli-BARToli, U., « Plasma diagnostics based on refractivity " in « Plasma Physics » Ed. International Atomic Energy Agency, Vienna (1965) p. 287.

[4] Helbig, V., Kelleher, D. E., Wiese, W. L., Phys. Rev. A 3 (1976) 1082.

[5] Baronnet, J. M., Rakowitz, J., Coudert, J. F., Bourdein, E., Fauchais, P., J. Phys. Colloq. France 40 (1979) C7 247.

[6] Bacri, J., Lagreca, M., J. Phys. D 16 (1983) 841.

[7] VAn De Hulst, H. C., Reesinck, J. J. M., Astrophys. J. 106 (1947) 121.
[8] Vacquie, S., Gleizes, A., Kafrouni, H., J. Phys. D 18 (1985) 2193.

[9] Schulz-Gulde, E., J. Phys. D 13 (1980) 793.

[10] Uhlenbusch, J. F., Fischer, E., Proc. IEEE 59 (1971) 578.

[11] KolesNiKov, V. N., «Spectroscopic investigations of non equilibrium plasma » invited paper XVI Int. Conf. Phen. Ionized Gases, Dusseldorf (1983) p. 45.

[12] Coudert, J. F., Fauchais, P., VII Int. Symp. Plasma Chem., Eindhoven (1985) p. 760.

[13] EdDy, T. L., J. Quant. Spectrosc. Radiat. Transfer. 33 (1985) 197.

[14] TaYlor, R. D., Ali, A. W., J. Quant. Spectrosc. Radiat. Transfer. 35 (1986) 213. 